\title{
Transition Effects of THz Helical Undulator Radiation in a Waveguide
}

\author{
B. Sargsyan \\ CANDLE Synchrotron Research Institute, Acharyan 31, 0040 Yerevan, Armenia \\ Email: bsargsyan@asls.candle.am
}

Received: November 4, 2021; Revised: November 22, 2021; Accepted: November 292021

\begin{abstract}
The radiation of a particle instantly injected into an infinite ideal cylindrical waveguide and implementing a helical motion is considered. Additionally, the problem of the radiation of a timevarying point charge moving stationary along a helical orbit in the same waveguide is also solved. Additionally, an explicit expression is obtained for the longitudinal component of the electric field of radiation with a wavefront moving together with the particle. The basic properties of radiation are determined: the conditions for its forward propagation are obtained, and its angular directivity is determined. A formula is given that describes radiation upon gradual introduction of a bunch into a waveguide (modeling the injection process) and during its subsequent propagation.
\end{abstract}

Keywords: Helical undulator, cylindrical waveguide, discrete radiation spectrum, time-varying point charge, injection process modeling

https://doi.org/10.54503/18291171-2021.14.4-165

\section{Introduction}

The helical undulator transforms its radiation spectrum in the presence of a waveguide, converting it from continuous to discrete, which significantly improves its radiation characteristics. This technique is especially effective in the terahertz frequency range. At a small radius of the waveguide, the spectral lines are located at a significant distance from each other, which makes it possible to use it in a single-frequency mode. By appropriate selection of undulator parameters, it is possible to achieve the concentration of most radiation energy in one of the waveguide modes [1], which will lead to its even more efficient use, maximizing the power of the emitted mode and suppressing the rest.

Except for works [2, 3], to date, the focus has been on the cases of stationary helical motion of a particle in infinite rectangular [4-6] and circular waveguides [1, 7-11]. Meanwhile, there is an injection process in real undulators, i.e., the introduction of a bunch into an undulator, which is equivalent to the gradual appearance of bunch particles in an undulator. When considering the helical motion of the bunch in the waveguide of finite dimensions, the deformations of the wavefront due to the principle of causality should be taken into account because of the temporary effects arising from the sudden appearance of a particle do not smooth out over time: the field amplitudes remain time-dependent.

In the present work, at first, as an auxiliary one, we consider the problem of the stationary motion of a point particle with a charge varying with time and performing a helical motion in an infinite ideal cylindrical waveguide. Then, on its basis, the problem of a particle that suddenly appears at a specific moment and moves along a helical trajectory in the same waveguide is solved. In conclusion, a formula is given that describes the gradual appearance of a bunch of charged particles, which simulates the process of its injection. 

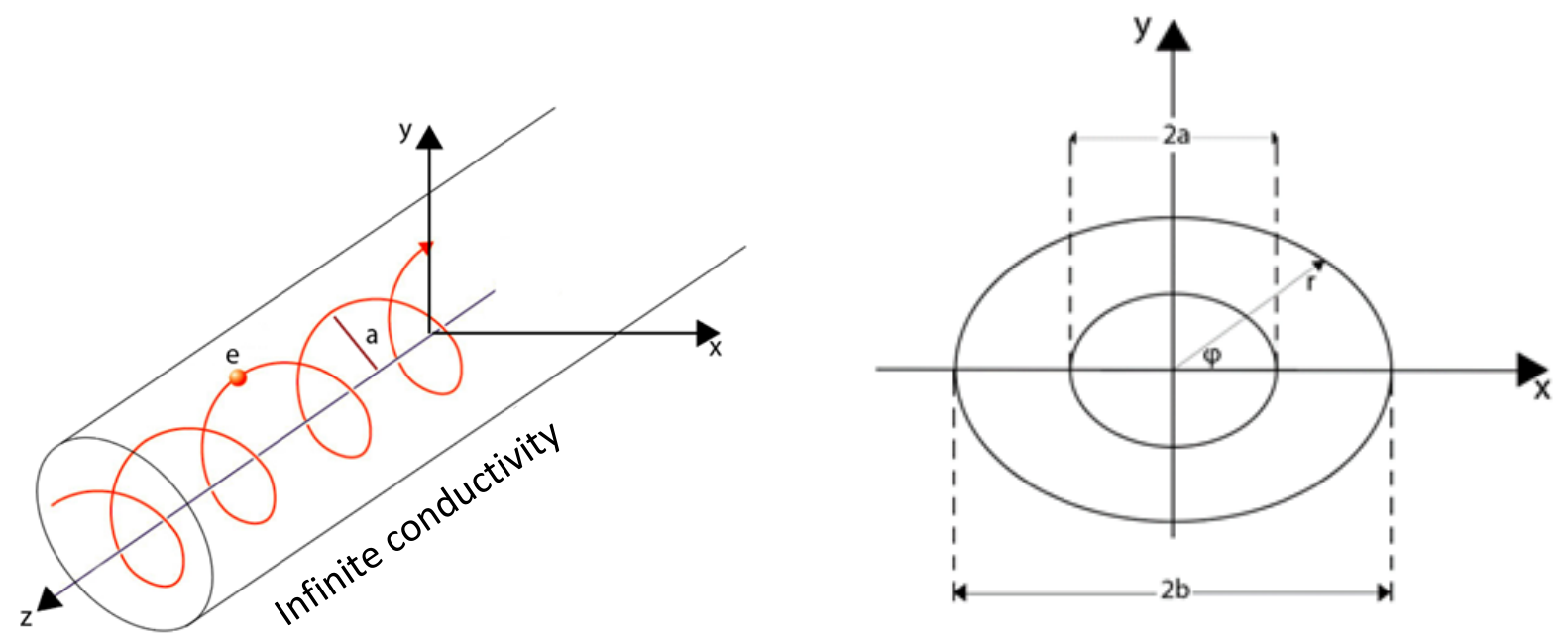

Fig. 1. The orbit of the helically moving charge and some parameters of the waveguide.

\section{Radiation of a charge varying in time}

Consider relativistic point charge with longitudinal velocity $\mathrm{V}$ and with the charge $Q(t)$, arbitrary dependent from the time, moving along the helical trajectory in the homogeneous waveguide. The motion takes place in the circular waveguide with the perfect conducting walls and the radius of $b$ (Fig. 1). The charge density $\tilde{\rho}$ and the charge current $\overrightarrow{\tilde{J}}$ are given in the forms:

$$
\begin{aligned}
& \rho(r, \varphi, z, t)=q Q(t) \frac{\delta(r-a)}{\sqrt{r a}} \delta\left(\varphi-\omega_{b} t\right) \delta(z-V t) \\
& \vec{\jmath}(r, \varphi, z, t)=q Q(t)\left(\omega_{b} a \vec{e}_{\varphi}+V \vec{e}_{z}\right) \frac{\delta(r-a)}{\sqrt{r a}} \delta\left(\varphi-\omega_{b} t\right) \delta(z-V t),
\end{aligned}
$$

where $\vec{e}_{\varphi}$ and $\vec{e}_{z}$ are unit vectors in the cylindrical coordinates and $V$ is the longitudinal velocity of the particle, $\omega_{0}=2 \pi V / \lambda_{u}$ is the particle revolution frequency, $\lambda_{u}$ is the undulator period. The helix radius $a$ can be given in the form of $a=\lambda_{u} K / 2 \pi \gamma$, where $K=0.93 B_{0}[T] \cdot \lambda_{u}[\mathrm{~cm}]$ is the dimensionless undulator parameter and $B_{0}$ is the maximum of the magnetic field on axial direction, $\gamma$ is the particle Lorentz factor.

The radiation fields are determined from the wave equations: The radiation fields are determined from the wave equations:

$$
\begin{aligned}
& \left\{\Delta-\frac{1}{c^{2}} \frac{\partial}{\partial t^{2}}\right\} \vec{E}=\vec{P} \\
& \left\{\Delta-\frac{1}{c^{2}} \frac{\partial}{\partial t^{2}}\right\} \vec{H}=\vec{Q}
\end{aligned}
$$

for the electrical and magnetic components of the radiation field, respectively.

Further:

$$
\begin{aligned}
& \vec{P}=\mu_{0}\left\{\frac{\partial \vec{\jmath}}{\partial t}+c^{2} \nabla \rho\right\} \\
& \vec{Q}=-\operatorname{rot} \vec{\jmath}
\end{aligned}
$$

The solutions of the wave equations (3) and (4) are sought by passing to the time-frequency domain: the right-hand sides of these equations are replaced by their Fourier transforms along the longitudinal coordinate $\mathrm{z}$ : 


$$
\vec{P}^{z}=e^{j k z} \int_{-\infty}^{\infty} \vec{P} e^{-j k z} d z, \vec{Q}^{z}=e^{j k z} \int_{-\infty}^{\infty} \vec{Q} e^{-j k z} d z
$$

or, explicitly:

$$
\begin{gathered}
\vec{P}_{r}^{z}=q Q(t) e^{j k(z-V t)} c^{2} \mu_{0} \delta^{\varphi}\left(-r^{-2} \delta(r-a)+r^{-1} \delta^{\prime}(r-a)\right) \\
\vec{P}_{\varphi}^{z}=q \mu_{0} \delta(r-a)\left\{\left(-j k V \omega_{0} \delta \delta^{\varphi}+r^{-2} c^{2} \delta^{\varphi^{\prime}}+\omega_{0}^{2} \delta^{\varphi^{\prime}}\right) Q(t)+\omega_{0} \delta^{\varphi} Q^{\prime}(t)\right\} e^{j k(z-V t)} \\
\vec{P}_{z}^{z}=q \mu_{0} \delta(r-a) r^{-1}\left\{\left(\delta^{\varphi} k\left(j c^{2}+V^{2}\right)+V \omega_{0} \delta^{\varphi^{\prime}}\right) Q(t)+V \delta^{\varphi} Q^{\prime}(t)\right\} e^{j k(z-V t)} \\
\delta^{\varphi}=\delta\left(\varphi-\omega_{0} t\right), \quad \delta^{\varphi^{\prime}}=\delta^{\prime}\left(\varphi-\omega_{0} t\right)
\end{gathered}
$$

The electrical and magnetic fields are sought in the form of vector cylindrical mode compositions, which combine TM and TE harmonics:

$$
\vec{E}=\sum_{n, m=1}^{\infty}\left\{\vec{E}_{n m}^{T M}+\vec{E}_{n m}^{T E}\right\}
$$

The longitudinal components of electric and magnetic harmonics are written in the form of expansion terms in Bessel functions of the first kind

$$
\begin{aligned}
& \tilde{E}_{n m_{z}}^{T M}=U_{n m} \psi_{n m}^{T M}, \quad \psi_{n m}^{T M}=J_{n}\left(j_{n m} \frac{r}{b}\right) e^{j n\left(\varphi-\omega_{0} t\right)} e^{j k(z-V t)} \\
& \widetilde{H}_{n m_{z}}^{T E}=c \varepsilon_{0} W_{n m} \psi_{n m}^{T E}, \quad \psi_{n m}^{T E}=J_{n}\left(v_{n m} \frac{r}{b}\right) e^{j n\left(\varphi-\omega_{0} t\right)} e^{j k(z-V t)}
\end{aligned}
$$

where $j_{n m}$ and $v_{n m}$ are the roots of the Bessel function and its derivative, respectively: $J_{n}\left(j_{n m}\right)=$ $0, J_{n}^{\prime}\left(v_{n m}\right)=0$.

Transverse components TM and TE of the radiation fields' frequency distributions are written in the form:

$$
\begin{array}{ll}
\vec{\varepsilon}_{n m}^{T M}=\left\{\varepsilon_{n m_{r}}^{T M}, \varepsilon_{n m_{\varphi}}^{T M}, 0\right\} & \overrightarrow{\mathcal{H}}_{n m}^{T E}=\left\{\mathcal{H}_{n m_{r}}^{T E}, \mathcal{H}_{n m_{\varphi}}^{T E}, 0\right\} \\
\overrightarrow{\widetilde{E}}_{n m_{t}}^{T M}=A_{n m} \vec{\varepsilon}_{n m}^{T M} & \overrightarrow{\widetilde{H}}_{t}^{T E}=D_{n m} \overrightarrow{\mathcal{H}}_{n m}^{T E} \\
\overrightarrow{\widetilde{H}}_{n m_{t}}^{T M}=C_{n m} c \varepsilon_{0}\left[\vec{e}_{z} \times \vec{\varepsilon}_{n m}^{T M}\right] & \overrightarrow{\vec{E}}_{t}^{T E}=-B_{n m}\left(c \varepsilon_{0}\right)^{-1}\left[\vec{e}_{z} \times \overrightarrow{\mathcal{H}}_{n m}^{T E}\right] \\
\varepsilon_{n m_{r}}^{T M}=\frac{\partial \psi_{n m}^{T M}}{\partial r}, \varepsilon_{n m_{\varphi}}^{T M}=j \frac{n}{r} \psi_{n m}^{T M} & \mathcal{H}_{n m_{r}}^{T E}=\frac{\partial \psi_{n m}^{T E}}{\partial r}, \mathcal{H}_{n m_{\varphi}}^{T E}=j \frac{n}{r} \psi_{n m}^{T E}
\end{array}
$$

Putting (9), (10) and (11) in the wave equation (3) give the system of equations for the amplitudes $U_{n m}, A_{n m}$ and $B_{n m}$ :

$$
\begin{aligned}
& \frac{e^{j k(z-V t)}}{b^{3} c^{2} r} \sum_{n, m=1}^{\infty} e^{j n\left(\varphi-\omega_{0} t\right)}\left\{j_{n m} r J_{n}^{\prime}\left(j_{n m} \frac{r}{b}\right) G_{A}+j b n J_{n}\left(v_{n m} \frac{r}{b}\right) G_{B}\right\}=P_{r}^{Z}, \\
& \frac{e^{j k(z-V t)}}{b^{3} c^{2} r} \sum_{n, m=1}^{\infty} e^{j n\left(\varphi-\omega_{0} t\right)}\left\{j b n J_{n}\left(j_{n m} \frac{r}{b}\right) G_{A}-v_{n m} r J_{n}^{\prime}\left(v_{n m} \frac{r}{b}\right) G_{B}\right\}=P_{\varphi}^{Z}, \\
& \frac{e^{j k(z-V t)}}{b^{2} c^{2}} \sum_{n, m=1}^{\infty} e^{j n\left(\varphi-\omega_{0} t\right)} J_{n}\left(j_{n m} \frac{r}{b}\right) G_{U}=P_{z}^{Z},
\end{aligned}
$$

Here

$$
\begin{aligned}
& G_{X}=f\left(g_{X}\right) X_{n m}+b^{2}\left(2 j \omega X_{n m}^{\prime}-X_{n m}^{\prime \prime}\right), X_{n m}=U_{n m}, A_{n m}, B_{n m}, \\
& f\left(g_{X}\right)=c^{2}\left(g_{X}^{2}+b^{2} k^{2}\right)-b^{2} \omega^{2}=b^{2}\left(\widetilde{\omega}^{2}-\omega^{2}\right), \widetilde{\omega}=\frac{c}{b} \sqrt{g_{X}^{2}+b^{2} k^{2}}, \\
& g_{n m}=j_{n m} \text { for } U_{n m} \text { and } A_{n m} \text { and } g_{n m}=v_{n m} \text { for } B_{n m} .
\end{aligned}
$$

Note the relationship between frequency $\omega$ and longitudinal wavenumber: $\omega=k v+n \omega_{0}$. 
Combining the obtained equations with each other and using the well-known orthogonality conditions for the Bessel functions [12], we obtain equations for the amplitudes $U_{n m}, A_{n m}, B_{n m}$ :

$$
G_{X}=f\left(g_{X}\right) X_{n m}+b^{2}\left(2 j \omega X_{n m}^{\prime}-X_{n m}^{\prime \prime}\right)=F_{X}\left(j K_{X} Q(t)+R_{X} Q^{\prime}(t)\right), X=U, A, B,
$$

where

$$
\begin{gathered}
\mathrm{F}_{U}=-q \mu_{0} \frac{c^{2}}{2 \pi^{2}} \frac{J_{n}\left(j_{n m} \frac{a}{b}\right)}{J_{n+1}^{2}\left(j_{n m}\right)}, \quad \mathrm{F}_{A}=-j q \mu_{0} \frac{c^{2}}{2 \pi^{2}} \frac{J_{n}\left(j_{n m} a / b\right)}{j_{n m}^{2} J_{n+1}^{2}\left(j_{n m}\right)}, \\
\mathrm{F}_{B}=-q \mu_{0} \frac{b a c^{2} \omega_{b}}{2 \pi^{2}} \frac{v_{n m}}{v_{n m}^{2}-n^{2}} \frac{J_{n}^{\prime}\left(v_{n m} a / b\right)}{J_{n}^{2}\left(v_{n m}\right)} \\
K_{U}=c^{2} k-V \omega, \quad R_{U}=V \\
K_{A} j_{n m}^{2}-b^{2} n \omega_{b} \omega, \quad R_{A}=b^{2} n \omega_{b} \\
K_{B}=\omega, R_{B}=-1
\end{gathered}
$$

For the amplitudes of the magnetic components of the field (wave equation (4)) equations are obtained with a structure identical to (14). In this case, we have:

$$
\begin{aligned}
& \mathrm{F}_{W}=j q \frac{2 a c \omega_{b}}{b \varepsilon_{0}} \frac{v_{n m}^{3}}{v_{n m}^{2}-n^{2}} \frac{J_{n}^{\prime}\left(v_{n m} a / b\right)}{J_{n}^{2}\left(v_{n m}\right)}, \quad K_{w}=1, \quad R_{W}=0, \quad g_{W}=v_{n m} \\
& \mathrm{~F}_{C}=j q \frac{2 c}{\varepsilon_{0}} \frac{j_{n m}^{2} V-b^{2} k n \omega_{b}}{J_{n-1}^{2}\left(j_{n m}\right)} \frac{J_{n m}\left(j_{n m} a / b\right)}{j_{n m}^{2}}, K_{C}=1, \quad R_{C}=0, \quad g_{C}=j_{n m} \\
& \mathrm{~F}_{D}=-q \frac{2 a b c k \omega_{b}}{\varepsilon_{0}} \frac{v_{n m}}{v_{n m^{2}}^{2} n^{2}} \frac{J_{n}^{\prime}\left(v_{n m} a / b\right)}{J_{n}^{2}\left(v_{n m}\right)}, K_{D}=1, R_{D}=0, \quad g_{W}=v_{n m}
\end{aligned}
$$

Equation (14) is a second order differential equation. Its complete solution can be composed of a particular solution of an inhomogeneous equation and a general solution of a homogeneous equation (with zero right-hand side).

The solution of the inhomogeneous equation (14) can be obtained by representing the amplitudes $X_{n m}$ and function $Q(t)$ through the images $\tilde{X}_{n m}$ and $\tilde{Q}$ of the Laplace transform in time:

$$
X_{n m}=\int_{0}^{\infty} \widetilde{U}_{n m} e^{-\alpha t} d \alpha, Q(t)=\int_{0}^{\infty} \tilde{Q} e^{-\alpha t} d \alpha
$$

The connection between the Laplace images of the amplitude and the function (15) is obtained after substituting (17) into (14) and equating the integrands in the left and right sides of the resulting equation:

$$
\tilde{X}_{n m}(\alpha)=\mathrm{F}_{X} \tilde{Q}(\alpha) P_{X}(\alpha)
$$

where

$$
P_{X}(\alpha)=\frac{j K_{X}-\alpha R_{X}}{f\left(g_{X}\right)-b^{2}\left(2 j \omega \alpha+\alpha^{2}\right)}
$$

The time dependence of the amplitude is determined using the inverse Laplace transform from the coordinate $\alpha$ to the time domain. Thus, for all six components, the solution has an identical form:

$$
X(t)=\mathrm{F}_{X} \mathcal{L}_{t}^{-1}\left\{\tilde{Q}(\alpha) P_{X}(\alpha)\right\}, \quad X=U, A, B, W, C, D
$$

An explicit solution can be obtained from (20) by substituting in it the Laplace image $\widetilde{Q}(\alpha)$ of a specific function $Q(t)$ of the time dependence function of charge accumulation. 


\section{The radiation of injected particle}

Now consider of the process of injection of the case of a single point particle arising at the time instant $t=0$ at the point $r=b, z=0, \varphi=0$ inside the waveguide and being drawn into motion along a helical trajectory by external magnetic fields. The phenomenon of instantaneous appearance of a point charged particle is described by the introduction of a step function into the expressions (1), (2) for charges and currents instead of function $Q(t): Q(t)=\chi(t)$, where $\chi(t)=0$ at $t<0$ and $\chi(t)=1$ at $t \geq 0$. The Laplace image of a step function is:

$$
\tilde{\chi}(\alpha)=\mathcal{L}_{\alpha}\{\chi(t)\}=\alpha^{-1}
$$

and the derivative of the step function at $t>0$ is equal to zero and $R_{X}=0$ in (19). Therefore, from (20) we have:

$$
X_{N}(t)=j \frac{\mathrm{F}_{N} K_{N}}{f\left(g_{N}\right)}\left\{1-e^{-j \omega t}\left(\cos \left(\widetilde{\omega}_{N} t\right)+j \frac{\omega}{\widetilde{\omega}_{N}} \sin \left(\widetilde{\omega}_{N} t\right)\right)\right\}
$$

The first term in (44) coincides with the expression for the stationary solution in an infinite waveguide

We represent the factor $b^{2} / f\left(g_{N}\right)$ in the form

$$
\frac{b^{2}}{f\left(g_{N}\right)}=u_{1}+u_{2}
$$

Here

$$
\begin{aligned}
& \frac{b^{2}}{f\left(g_{N}\right)}=u_{0}=\frac{1}{\left(k_{1}-k_{2}\right)\left(k-k_{1}\right)}-\frac{1}{\left(k_{1}-k_{2}\right)\left(k-k_{2}\right)} \\
& u_{1}=\frac{k}{k_{1}\left(k_{1}-k_{2}\right)\left(k-k_{1}\right)}-\frac{1}{k_{2}\left(k_{1}-k_{2}\right)\left(k-k_{2}\right)} \\
& u_{2}=-\frac{1}{k_{1}\left(k_{1}-k_{2}\right)}+\frac{1}{k_{2}\left(k_{1}-k_{2}\right)}=\frac{1}{k_{1} k_{2}} \\
& k_{1,2}=\frac{b V n \omega_{0} \pm c \sqrt{b^{2} n^{2} \omega_{0}^{2}-g_{N}^{2}\left(c^{2}-V^{2}\right)}}{b\left(c^{2}-V^{2}\right)}
\end{aligned}
$$

$k_{1,2}$ are the roots of equation $f\left(g_{N}\right)=0$ with respect to $k$.

Now (22) can be rewritten as:

with

$$
X_{N}(t)=j \mathrm{~F}_{N} K_{N} u_{0}-e^{-j \omega t} Z(k) u_{1}-e^{-j \omega t} Z(k) u_{2}+e^{-j \omega t} Z_{0}(k)
$$

$$
\begin{aligned}
& Z(k)=j \mathrm{~F}_{N} K_{N}\left(\cos \left(\widetilde{\omega}_{N} t\right)+j \frac{\omega}{\widetilde{\omega}_{N}} \sin \left(\widetilde{\omega}_{N} t\right)\right) \\
& Z_{0}(k)=\left(A_{0}(k) \cos \left(\widetilde{\omega}_{N} t\right)+B_{0}(k) \sin \left(\widetilde{\omega}_{N} t\right)\right)
\end{aligned}
$$

In (25), a general solution of the homogeneous equation for amplitudes (with zero right-hand side)

$$
G_{X}=f\left(g_{X}\right) X_{n m}+b^{2}\left(2 j \omega X_{n m}^{\prime}-X_{n m}^{\prime \prime}\right)=0
$$

with indefinite so far coefficients $A_{0}(k)$ and $B_{0}(k)$ is added.

The transition to the space-time domain is accomplished using the inverse Fourier transform versus $k$ : 


$$
X_{N}(t)=X_{N}^{(0)}(t)+X_{N}^{(1)}(t)+X_{N}^{(2)}(t)+X_{N}^{(3)}(t)
$$

with

$$
\begin{aligned}
& X_{N}^{(0)}(t)=j \mathrm{~F}_{N} \int_{-\infty}^{\infty} K_{N} u_{0} e^{j(\omega t-k z)} d k, \quad X_{N}^{(1)}(t)=\int_{-\infty}^{\infty} Z(k) u_{1} e^{-j k z} d k \\
& X_{N}^{(2)}(t)=\int_{-\infty}^{\infty} Z(k) u_{2} e^{-j k z} d k, \quad X_{N}^{(3)}(t)=\int_{-\infty}^{\infty} Z_{0}(k) e^{-j k z} d k
\end{aligned}
$$

The integrand of $X_{N}^{(0)}(t)$ is an analytic function on the entire complex plane $k$ and, with the exception of points $k=k_{1}$ and $k=k_{2}$, corresponding to two simple poles lying on the real axis. Its value (in the sense of the principal value) is determined by the residues of these poles in the usual way [7]. The integrand of $X_{N}^{(1)}(t)$ has the same poles, but it is not an analytic function. The results of integration along the edges of the cuts emanating from the branch points $k= \pm j g_{N} / b$ should be also added to the contributions from the poles to its value. Asymptotically this contribution can be calculated by the saddle point or stationary phase method [13] It is easy to see that the contribution from the poles to the integral $X_{N}^{(1)}(t)$ completely vanishes the integral $X_{N}^{(0)}(t)$, and the additional contribution of the integral $X_{N}^{(1)}(t)$ (calculated, for example, by the stationary phase method), is compensated by the appropriate selection of the amplitudes $A_{0}(k)$ and $B_{0}(k)$ in the integral $X_{N}^{(3)}(t)$. Only the third term $X_{N}^{(2)}(t)$ in (28) remains nonzero. It does not contain poles, but its integrand is not an analytic function either. This integral

$$
X_{N}^{(2)}(t)=\frac{j \mathrm{~F}_{U}}{k_{1} k_{2}} \int_{-\infty}^{\infty} K_{U}\left(\cos \left(\widetilde{\omega}_{N} t\right)+j \frac{\omega}{\widetilde{\omega}_{N}} \sin \left(\widetilde{\omega}_{N} t\right)\right) e^{-j k z} d k
$$

can be calculated explicitly. In particular, for the longitudinal electric component $E_{z}$ we have:

$$
E_{z}=\frac{j \mathrm{~F}_{U}}{k_{1} k_{2}}\left\{\mathcal{M}_{0} J_{0}\left[\frac{j_{n m}}{b} \sqrt{c^{2} t^{2}-z^{2}}\right]+\mathcal{M}_{1} J_{1}\left[\frac{j_{n m}}{b} \sqrt{c^{2} t^{2}-z^{2}}\right]\right\}
$$

for $c^{2} t^{2}>z^{2}$ and $E_{z}=0$ for $c^{2} t^{2}<z^{2}$. The latter is due to the time delay of radiation.

Where,

$$
\begin{gathered}
\mathcal{M}_{0}=-j \frac{\pi}{c}\left\{V n^{2} \omega_{0}^{2}-z\left(c^{2} t+j_{n m} V z\right) \frac{j_{n m}\left(c^{2}-V^{2}\right)}{b^{2}\left(c^{2} t^{2}-z^{2}\right)}\right\} \\
\mathcal{M}_{1}=\frac{\mathfrak{R}_{1}+j \mathfrak{R}_{2}}{b c\left(c^{2} t^{2}-z^{2}\right)^{3 / 2}} \\
\mathfrak{R}_{1}=n \pi \omega_{0}\left(c^{2} t^{2}-z^{2}\right)\left(V\left(j_{n m} V z+c^{2} t\right)-z j_{n m}\left(c^{2}-V^{2}\right)\right) \\
\mathfrak{R}_{2}=-\pi\left(c^{2}-V^{2}\right)\left(z\left(j_{n m} V z+c^{2} t\right)+c^{2} t\left(j_{n m} V t+z\right)\right)
\end{gathered}
$$

During of calculation (31) the following relation [14] was used

$$
\int_{0}^{\infty} \frac{\sin \left[b \sqrt{a^{2}+x^{2}}\right]}{\sqrt{a^{2}+x^{2}}} \cos (x y) d x=\frac{1}{2}\left\{\begin{array}{c}
\pi J_{0}\left[a \sqrt{b^{2}-y^{2}}\right], \quad 0<y<b \\
0, \quad b<y<\infty
\end{array}\right.
$$

In contrast to the case of homogeneous motion in infinite waveguide, in the case under consideration, the waveguide is filled with energy as the particle deepens into the waveguide. The front of the propagated wave is determined by equality $c t=z$. Note that the field component (31) at $c t \rightarrow z$ tends to a finite limit. 
For the presence of radiation at a certain observation point $r, \varphi, z$ inside the waveguide, as already noted, the principle of causality must be observed, which requires the following relations to be satisfied:

$$
\frac{l_{1}}{V}+\frac{l_{2}}{c}=t, l_{1}+l_{2} \cos \alpha=z
$$

Here $l_{1}$ is the distance along the $\mathrm{z}$ axis, indicating the position of the particle at a certain moment of time $t^{\prime}<t$, at which its radiation reaches the point of observation $r, \varphi, z$ at the moment of time $t$. $l_{2}$ denotes the distance between the particle and the point of observation, which is passed by the radiation emanating from the particle at time $t^{\prime}$ and reaching the point of observation at time $t ; \alpha$ is the angle between the line connecting the particle and the observation point and the axis of the waveguide.

From (52) it follows:

$$
l_{1}=\frac{V(c t \cos \alpha-z)}{c \cos \alpha-V}, l_{2}=\frac{c(z-t V)}{c \cos \alpha-V}
$$

Important conclusions follow from (35) under conditions $l_{1,2}>0$ : (a) forward radiation is possible if $z>t V$, (b) the radiation is concentrated near the axis of the waveguide within the conical angle $\alpha \leq \min \left\{\sqrt{2 /\left(1-V^{2} / c^{2}\right)}, \sqrt{1-(z / c t)^{2}}\right\}$.

Frequency characteristics of radiation can be determined by analyzing the integrand in formula (30). For an arbitrary value of the function $f\left(j_{n m}\right)$, it is a rapidly oscillating function, while with $f\left(j_{n m}\right)=0$ the oscillations remain only in phase and its modulus slowly varied with frequency. For this reason, its frequency distribution is characterized by sharp peaks at frequencies determined by equation $f\left(j_{n m}\right)=0$. Thus, the resonant frequencies remain the same as in the stationary motion of a particle in an infinite waveguide [7]. Obtained formula (31) describes narrow-directed and narrow-band radiation. The process of the emergence and subsequent propagation of an arbitrary bunch of uniform length $t_{0}$ can be described using the convolution of the expression for the field of a point particle (31) with the charge longitudinal distribution function $f_{z}(t)$ in the bunch:

$$
E_{z}^{b}(t)=\int_{0}^{t_{u}} f_{z}\left(t-t^{\prime}\right) E_{z}\left(t^{\prime}\right) d t^{\prime}
$$

Here $t_{u}=t$ at the formation of a bunch and $t_{u}=t_{0}$ at its completion.

For brevity, we obtained an explicit expression only for the longitudinal electrical component (31). Similarly, (using formulas (15) and (16)), the rest of the components can be calculated.

\section{Conclusions}

The results of this work make it possible to study in more detail the processes of emission of bunches in a helical undulator combined with waveguide, occurring during injection, subsequent propagation, and after leaving the open end (open cross-section) of the waveguide. They will contribute to the creation of mathematical models of the operation of an undulator-waveguide structure close to reality. The results related to the time-varying charge of a particle can find application, for example, when taking into account the loss of particles in a bunch arising from scattering on the walls of the waveguide and scattering on molecules of the residual gas. 


\section{Acknowledgements}

The work was supported by the Science Committee of MESCS RA, in the frames of the research project № 21T-1C239.

\section{References}

[1] M.I. Ivanyan, A. Grigoryan, B. Sargsyan, T. Vardanyan, V.M. Tsakanov, A.V. Tsakanian, Laser Phys. 30 (2020) 115002.

[2] M.L. Levin, ZhTF 17 (1947) 1159.

[3] G.G. Karapetian, Izv. Akad. Nauk Arm. SSR, Fiz. 12 (1977) 186.

[4] H.A. Haus, N. Islam, J. Appl. Phys. 54 (1983) 9.

[5] A. Amir, I. Boscolo, L.R. Elias, Phys. Rev A 32 (1985) 5.

[6] Y. H. Chin, Fourth Advanced ICFA Beam Dynamics Workshop, Report LBL-29981 (1990).

[7] V.P. Dokuchaev, Izv. VUZov, Radiofizika 44 (2001) 587.

[8] A.S. Kotanjyan, A.A. Saharyan, J. Phys. A 40 (2007) 10641.

[9] G. Geloni, E. Saldin, E. Schneidmiller, M. Yurkov, Nucl. Instr. and Methods in Phys. Research A, 584 (2008) 219.

[10] T. Vardanyan, A. Grigoryan, L. Hovakimyan, M. Ivanyan, A. Tsakanian, V. Tsakanov, Proc. FEL2014 (2014) 26.

[11] M. Ivanyan, A. Tsakanian, T.Vardanyan, Arm. J. Phys. 8 (2015) 56.

[12] B.M. Kincaid, J. Appl. Phys. 48 (1977) 2684.

[13] M. Born, E. Wolf, Principles of Optics (Cambridge University Press, Cambridge, 2013).

[14] H. Bateman, A. Erdelyi, Tables of Integral Transforms, 1 (McGRAW-HILL Book Company, INC. USA, 1954). 